\title{
Impact of non-oncological and oncological factors on tumor recurrence in HCC liver transplanted patients-single liver transplant centre experience
}

\author{
Gabriela Smira1 , Vladislav Brasoveanu², Simona Ichim', Florin Ichim², \\ Razvan Grigorie'2, Mihail Pautov², Doina Hrehoret ${ }^{2}$, Irinel Popescu² \\ ${ }^{1}$ Center of Gastroenterology and Hepatology, Fundeni Clinical Institute, Bucharest, Romania \\ ${ }^{2}$ Center of General Surgery and Liver Transplantation, Fundeni Clinical Institute, Bucharest, Romania
}

\begin{abstract}
Background. The aim of this study was to identify pre-transplant risk factors that lead to tumor recurrence and to evaluate mortality in liver transplanted patients from the single Liver Transplant Center in Romania. Methods. 81 patients who underwent liver transplantation between 2011 and 2015 at the Fundeni Clinical Institute were assessed based on age, sex, type of operation, histopathological staging, pre-transplant bridging therapies, etiology of cirrhosis, San Francisco and Up to seven scores, AFP and CA19-9.

Results. Between 2011 and 2015, 81 out of 454 liver transplants were performed for hepatocarcinoma (17.84\%). The most frequent etiology was the HVB and HVD co-infection $39.7 \%$. The 1 and 3 years-survival rates were $81.15 \%$, respectively $72.5 \%$. The 1 year and 3 years tumor recurrence rate were $14.49 \%$, respectively 15.

Conclusions. We identified as risk factors for 1 year-tumor recurrence the following: pre-transplant Milan, San Francisco and Up to Seven scores, pre-transplant AFP > $200 \mathrm{ng} / \mathrm{ml}$ and Edmonson/Steiner staging. Age over 65 at transplant was not correlated to cancer recurrence, 1 year and 3 years mortality rates or postoperative complications.
\end{abstract}

Keywords: hepatocellular carcinoma, liver transplantation, risk factors, tumor recurrence

\section{INTRODUCTION}

HCC constitutes the fifth most frequent form of cancer worldwide and it holds the second place in malignancy-related mortality. HCC occurs two to six times more frequently in men than in women(1).The key risk for HCC is liver cirrhosis, approximately $80 \%$ of which are related to hepatitis B and C.In Eastern Europe, Romania is on the first place in hepatocarcinoma related mortalityand second regarding liver cancer incidence (2).

Chronic hepatitis B is the major risk factor for developing HCC in Africa and Asia, while in the US, Europe and Japan chronic hepatitis C, alcohol and non-alcoholic steatohepatitis (NASH) are leading causes of HCC $(6,7)$. Eighty percent of liver cancers are found in cirrhotic livers, which themselves carry a high risk for HCC. Chronic carriers of hepatitis B virus (HBV) have a 100-fold increased risk as compared to a non-infected healthy reference population.There is growing evidence that the hepatitis B virus (HBV) contributes to hepatocarcinogenesis via direct malignant transformation and other indirect effects. Furthermore, persistent HBV infection can increase genetic instability by causing hepatocyte destruction and regeneration.

Moreover, HBV load is involved in post-LT HCC recurrence and increases the risk of post-LT recurrence through an inflammatory effect after HBV or hepatitis $\mathrm{C}$ virus (HCV) allograft re-infection $(5,8)$. Li et al. retrospectively analyzed 340 HBV-positive patients who underwent orthotropic LT (OLT) and found that HBV relapse was an independent predictor of $\mathrm{HCC}$ recurrence $(\mathrm{P}=0.03)$, 
and that high pre-transplant levels of HBV DNA were associated with $\mathrm{HCC}$ recurrence. $\mathrm{Wu}$ et al. also performed a retrospective study of 78 patients with HBV-related HCC who underwent LT, and found that 13 patients $(16.6 \%)$ experienced $\mathrm{HCC}$ recurrence and 18 patients $(23.1 \%)$ experienced HBV relapse. Therefore, the authors concluded that HBV relapse was closely related to HCC recurrence $(\mathrm{P}=0.004)$ and led to a shorter OS after LT. Thus, HBV relapse and HCC recurrence may have a reciprocal causative relationship in the post-transplantation setting .

Approximately 130-170 million people globally are infected with the hepatitis $\mathrm{C}$ virus (HCV), 20 to $30 \%$ of whom will develop liver cirrhosis which carries a $3-5 \%$ annual risk of ultimately progressing to liver cancer. Unlike HBV, there is no close relationship between $\mathrm{HCV}$ and RNA and the risk of developing HCC. As a general rule, patients will not develop liver cancer in chronic $\mathrm{HCV}$ before their disease has progressed to advanced fibrosis and cirrhosis. The risk of HCV-induced HCC appears to be related to the degree of inflammation and necrosis, while HBV-related HCC does not correlate well with inflammation and seems rather to involve activation of specific oncogenes by the virus.

The need for LT exceeds the number of deceased donors, which increases waiting times and contributes to a high drop-out rate among patients who experience tumor progression while awaiting surgery $(9,11)$. Thus, living donor LT (LDLT) provides patients with HCC better access totimely treatment. However, several recent studies have demonstrated that LDLT is associated with an increased incidence of post-LT HCC recurrence, compared to deceased donor LT (DDLT) (11-13).

There are three suggested mechanisms by which LDLT might increase the risk of HCC recurrence. The first mechanism is the release of growth factors that mediate the regeneration of the hemiliver and increase the vascular inflow during the rapid regeneration of the partial grafts from living donors, which might contribute to tumor progression and recurrence. Furthermore, small-sized grafts are more likely to cause acute phase graft injury, which results in cell adhesion, angiogenesis, and migration; all of these factors may promote tumor recurrence $(14-16,17)$.

The second mechanism is the "fast-tracking effect", whereby patients who undergo LDLT have a shorter waiting time, which might preclude the detection of an aggressive tumor before surgery and increase the risk of recurrence $(18,19)$.
The third mechanism is the LDLT technique itself might directly contribute to a higher recurrence rate, due to the sparing of the inferior vena cava (which is necessary for complete tumor removal) and more extensive liver manipulation during the LDLT. All of these factors might contribute to the high recurrence rates after LDLT compared to after DDLT $(12,20)$.

Despite these potential mechanisms by which LDLT might increase HCC recurrence, other studies have reported that LDLT recipients have a similar recurrence rate and comparable recurrence-free survival compared to patients who underwent DDLT. In these studies, the authors attributed the inferior outcomes after LDLT for HCC to the tumor's characteristics and biology. Although there is no clear evidence regarding whether LDLT is associated with a higher recurrence rate, the conflicting data suggest that different indication criteria may be appropriate for LDLT and DDLT (21-23).

In general, there is an arbitrary age limit for LT, due to the increased incidence of age-related comorbidities among elderly patients with HCC $(24,25)$.Several studies have reported that elderly patients who underwent LT exhibited a lower survival rate and higher rates of HCC malignancy, which may be associated with their increased risk of adverse outcomes due to chronic comorbidities, immunosuppression, and immunosenescence. $(25,26)$. Age-related immunological changes and immunosenescence can increase the susceptibility of elderly patients to infection, autoimmune disease, and cancer; and long-term immunosuppressive therapy after LT might increase these patients' risks of morbidity and mortality compared to their younger counterparts. However, other studies have reported that LT is not contraindicated for elderly patients, and Ballarin et al. reported similar shortand middle-term survival outcomes and morbidities (e.g., HCC recurrence) among young and elderly patients.

Moreover, Kim et al. demonstrated that OS was prolonged among younger patients who underwent OLT for HCC, although there were no significant differences in HCC-specific survival among the various age groups (27).Therefore, these findings suggest that carefully selected elderly patients with HCC could experience a benefit from OLT that is equal to the benefit that is experienced by younger patients.

Alpha-fetoprotein (AFP) is a serum tumor marker for hepatocellular carcinoma (HCC) and can also be used to monitor HCC recurrence after liver transplantation (LT). Although AFP can be slightly 
elevated in several other conditions, such as chronic hepatitis and liver cirrhosis, high preoperative levels of AFP are associated with poorer outcomes following liver transplantation for $\operatorname{HCC}(5,6,7)$. Recently, the significance of AFP decline after curative resection for $\mathrm{HCC}$ has been reported as a predictor of tumor recurrence $(8,9)$. Nobuoka et al. (9) emphasized that a positive level of AFP within 4 months after resection was a strong predictor of post-operative tumor recurrence. Little is known, however, about the relationship between peri-operative change in AFP level and tumor recurrence or the clinical usefulness of peri-operative change as an early marker of $\mathrm{HCC}$ recurrence.

Based on the studies in resection, we hypothesized that a rapid decline in AFP may be associated with better recurrence-free survival (RFS) in patients who have undergone LT.

This study, therefore, evaluated the clinical significance of change in peri-transplant serum AFP level as a predictor of tumor recurrence in $\mathrm{HCC}$ patients who underwent liver transplantation.

The aim of this study was to identify pre-transplant risk factors that lead to tumor recurrenceand to evaluate mortality in liver transplanted patients from the single Liver Transplant Center in Romania.

\section{MATERIALS AND METHODS}

81 patients who underwent liver transplantation between 2011 and 2015 at Fundeni Clinical Institute were assessed based on age, sex, type of operation performed, histopathological staging, pretransplant bridging therapies, etiology of cirrhosis, San Francisco and Up to seven scores, AFP and CA19 9 values before and at 1, 3, 6 and 12 months post-transplant. The prevalence of cirrhosis among patients with hepatocellular carcinoma has been 95\% and the HCC incidence rate among patients with cirrhosis has been shown to be $2-4 \%$ per year.

\section{RESULTS}

Between 2011 and 2015, 81 out of 454 liver transplants were performed for hepatocarcinoma (17.84\%), 26 of them received living related liver transplantation. The most frequent etiology was the HVB and HVD co-infection $39.7 \%$, followed in close range by the HVC infection $25.4 \%$ and alcoholism $22.2 \%$ (Fig. 1). The median follow-up period was 36 months. The 1 year and 3 years-survival rates were $81.15 \%$, respectively $72.5 \%$ (Fig. 2). No death after the first year post liver transplant was associated to tumor recurrence. The 1 year and 3 years tumor recurrence rate were $14.49 \%$, respectively $15.94 \%$. Milan, San Francisco and Up to Seven scores, pre-transplant AFP > $200 \mathrm{ng} / \mathrm{ml}$ and Edmonson/Steiner staging were found to be statistically significant associated to tumor recurrence $(\mathrm{p}=0.01 ; \mathrm{p}=0.00032 ; \mathrm{p}=0.01)$ (Fig. 3).

Age over 65 years was not significantly correlated to tumor recurrence, mortality or postoperative complications $(\mathrm{p}=0.2 ; \mathrm{p}=0.37 ; \mathrm{p}=0.8)$.
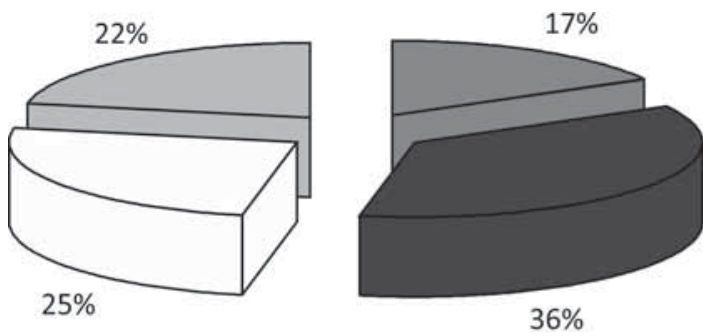

HBV and HDV Coinfection

$\square$ HCV Infection

Alcoholism

Others

FIGURE 1. The Etiology of Cirrhosis

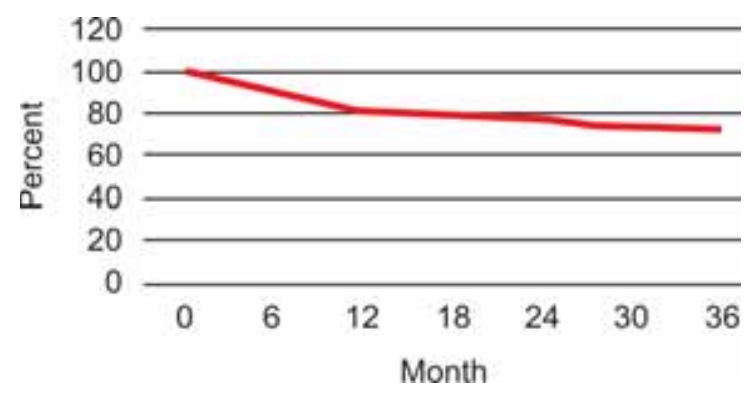

FIGURE 2. Survival rates after $L T$

\section{DISCUSSION}

Liver transplantation has been shown to be the best therapeutic option in selected patients with HCC. Although transplants are usually performed in patients who meet the Milan criteria, a substantial subset of patients who do not meet these criteria have the potential for good outcomes after liver transplantation, leading to the proposal of several new criteria $(5,10)$. These latter criteria emphasize the importance of the inclusion of new markers of biological behavior, along with morphological tumor size and number, in formulating new sets of criteria that can better predict the risk of recurrence. AFP is one of strong biological markers and used for early diagnosis of HCC and for recurrence after LT $(28,29)$. Numerous study groups have empha- 


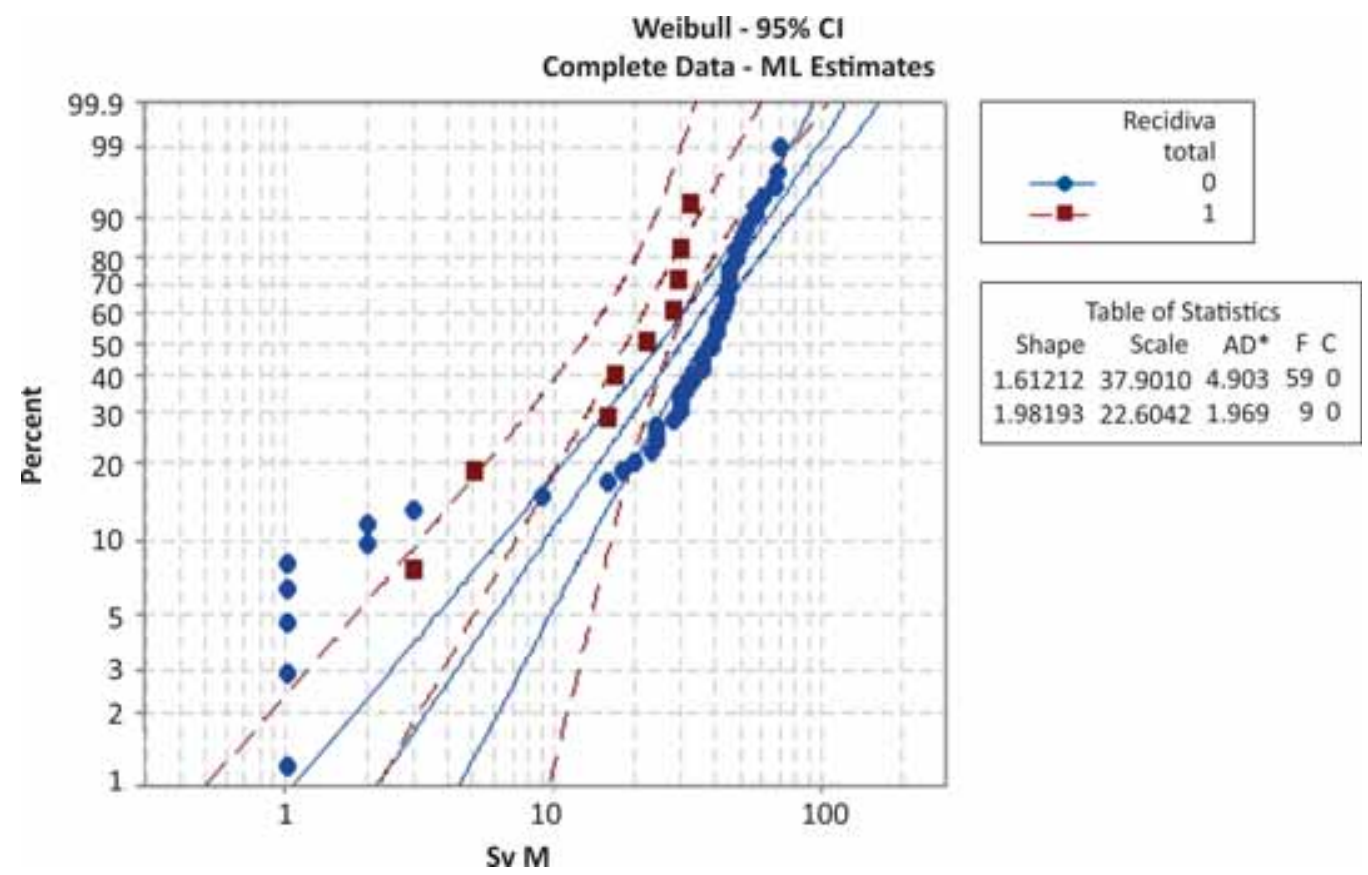

FIGURE 3. Probability Plot for Sv M

sized that high preoperative levels of AFP are associated with poorer outcomes from liver transplantation for HCC.

Pre-LT high AFP is an important risk factor for HCC recurrence (30).

Patients undergoing liver transplantation for hepatocellular carcinoma within the Milan criteria ( single tumour $\leq 5 \mathrm{~cm}$ in size or $\leq 3$ tumours each $\leq 3$ $\mathrm{cm}$ in size, and no macrovascular invasion) have an excellent outcome. Age over 65 at transplant was not correlated to cancer recurrence.

In conclusion, pre-transplant AFP is significantly prognostic of tumor recurrence after liver transplantation for HCC in patients with elevated pre-LT AFP level.(28-30).

\section{CONCLUSIONS}

Our results about survival and cancer-free survival at one year post-transplant correspond to specialty literature $(81.15 \%$ vs. $82 \%) /(84.06 \%$ vs. $80 \%$ ). We identified as risk factors for 1 year-tumor recurrence the following: pre-transplant Milan, San Francisco and Up to Seven scores, pre-transplant AFP $>200 \mathrm{ng} / \mathrm{ml}$ and Edmonson/Steiner staging. Age over 65 at transplant was not correlated to cancer recurrence.

1 year and 3 years mortality rates or postoperative complications and that supports the current European trend to discard this variable during the liver graft allocation process.

\section{REFERENCES}

1. Sotiropoulos GC, Molmenti EP, Lösch C, Beckebaum S, Broelsch CE, Lang $\mathrm{H}$. Meta-analysis of tumor recurrence after liver transplantation for hepatocellular carcinoma based on 1,198 cases. Eur J Med Res. 2007.

2. Ishikawa T. Anti-viral therapy to reduce recurrence and improve survival in hepatitis B virus-related hepatocellular carcinoma. World $\mathrm{J}$ Gastroenterol. 2013.

3. Li MR, Chen GH, Cai CJ, Wang GY, Zhao H. High hepatitis B virus DNA level in serum before liver transplantation increases the risk of hepatocellular carcinoma recurrence. Digestion. 2011.

4. Saab S, Yeganeh M, Nguyen K, Durazo F, Han S, Yersiz H, Farmer DG, Goldstein LI, Tong MJ, Busuttil RW. Recurrence of hepatocellular carcinoma and hepatitis $B$ reinfection in hepatitis B surface antigenpositive patients after liver transplantation. Liver Transpl. 2009.

5. Park SJ, Freise CE, Hirose R, Kerlan RK, Yao FY, Roberts JP, Vagefi PA. Risk factors for liver transplant waitlist dropout in patients with hepatocellular carcinoma. Clin Transplant. 2012;26.

6. Bittermann T, Niu B, Hoteit MA, Goldberg D. Waitlist priority for hepatocellular carcinoma beyond milan criteria: A potentially appropriate decision without a structured approach. Am J Transplant. 2014;14:79-87.

7. Park MS, Lee KW, Suh SW, You T, Choi Y, Kim H, Hong G, Yi NJ, Kwon $\mathrm{CH}$, Joh JW et al. Living-donor liver transplantation associated with higher incidence of hepatocellular carcinoma recurrence than deceased-donor liver transplantation. Transplantation. 2014.

8. Grant RC, Sandhu L, Dixon PR, Greig PD, Grant DR, McGilvray ID. Living vs. deceased donor liver transplantation for hepatocellular carcinoma: A systematic review and meta-analysis. Clin Transplant. 2013.

9. Vakili K, Pomposelli JJ, Cheah YL, Akoad M, Lewis WD, Khettry U, Gordon F, Khwaja K, Jenkins R, Pomfret EA. Living donor liver transplantation for hepatocellular carcinoma: Increased recurrence but improved survival. Liver Transpl. 2009;15. 
10. Man K, Lo CM, Xiao JW, Ng KT, Sun BS, Ng IO, Cheng Q, Sun CK, Fan ST. The significance of acute phase small-for-size graft injury on tumor growth and invasiveness after liver transplantation. Ann Surg. 2008;247.

11. Shi JH, Huitfeldt HS, Suo ZH, Line PD. Growth of hepatocellular carcinoma in the regenerating liver. Liver Transpl. 2011;17:866-874.

12. Yang ZF, Poon RT, Luo Y, Cheung CK, Ho DW, Lo CM, Fan ST. Up-regulation of vascular endothelial growth factor (VEGF) in small-for-size liver grafts enhances macrophage activities through VEGF receptor 2-dependent pathway. J Immunol. 2004;173:25072515.

13. Man K, Fan ST, Lo CM, Liu CL, Fung PC, Liang TB, Lee TK, Tsui SH, $\mathrm{Ng} \mathrm{IO}$, Zhang ZW et al. Graft injury in relation to graft size in right lobe live donor liver transplantation: A study of hepatic sinusoidal injury in correlation with portal hemodynamics and intragraft gene expression. Ann Surg. 2003;237:256-264.

14. Kulik L, Abecassis M. Living donor liver transplantation for hepatocellular carcinoma. Gastroenterology. 2004.

15. Fisher RA, Kulik LM, Freise CE, Lok AS, Shearon TH, Brown RS, Ghobrial RM, Fair JH, Olthoff KM, Kam I et al. Hepatocellular carcinoma recurrence and death following living and deceased donor liver transplantation. Am J Transplant. 2007.

16. Di Sandro S, Slim AO, Giacomoni A, Lauterio A, Mangoni I, Aseni P, Pirotta V, Aldumour A, Mihaylov P, De Carlis L. Living donor liver transplantation for hepatocellular carcinoma: Long-term results compared with deceased donor liver transplantation. Transplant Proc. 2009.

17. Xiao GQ, Song JL, Shen S, Yang JY, Yan LN. Living donor liver transplantation does not increase tumor recurrence of hepatocellular carcinoma compared to deceased donor transplantation. World J Gastroenterol. 2014.

18. Sandhu L, Sandroussi C, Guba M, Selzner M, Ghanekar A, Cattral MS, McGilvray ID, Levy G, Greig PD, Renner EL et al. Living donor liver transplantation versus deceased donor liver transplantation for hepatocellular carcinoma: comparable survival and recurrence. Liver Transpl. 2012;18:315-322

19. Bhangui P, Vibert E, Majno P, Salloum C, Andreani P, Zocrato J, Ichai P, Saliba F, Adam R, Castaing D et al. Intention-to-treat analysis of liver transplantation for hepatocellular carcinoma: living versus deceased donor transplantation. Hepatology. 2011;53:1570-1579.
20. Taner CB, Ung RL, Rosser BG, Aranda-Michel J. Age is not a contraindication for orthotopic liver transplantation: A single institution experience with recipients older than 75 years. Hepatol Int. 2012;6:403-407.

21. Herrero JI, Lucena JF, Quiroga J, Sangro B, Pardo F, Rotellar F, Alvárez-Cienfuegos J, Prieto J. Liver transplant recipients older than 60 years have lower survival and higher incidence of malignancy. Am J Transplant. 2003;3:1407-1412.

22. Malinis MF, Chen S, Allore HG, Quagliarello VJ. Outcomes among older adult liver transplantation recipients in the model of end stage liver disease (MELD) era. Ann Transplant. 2014;19:478-487

23. Kim J, Ko ME, Nelson RA, Arrington A, Luu C, Falor AE, Nissen NN, Colquhoun S, Hurria A, Singh G. Increasing age and survival after orthotopic liver transplantation for patients with hepatocellular cancer. J Am Coll Surg. 2014;218:431-438.

24. Poon D et al. Management of hepatocellular carcinoma in Asia: Consensus statement from the Asian Oncology Summit 2009. The Lancet Oncology. 2009;10:1111-1118

25. 2El-Serag HB, Kanwal F. Alpha-Fetoprotein in hepatocellular carcinoma surveillance: mend it but do not end it. Clin Gastroenterol Hepatol. 2013;11:441-443.

26. Lee E, Edward S, Singal AG, Lavieri MS, Volk M. Improving screening for hepatocellular carcinoma by incorporating data on levels of alpha-fetoprotein, over time. Clin Gastroenterol Hepatol. 2013;11:437440.

27. Blank $S$ et al. Assessing prognostic significance of preoperative alpha-fetoprotein in hepatitis B-associated hepatocellular carcinoma: normal is not the new normal. Ann Surg Oncol. 2014;21:986-994.

28. An SL et al. Prognostic Significance of Preoperative Serum Alphafetoprotein in Hepatocellular Carcinoma and Correlation with Clinicopathological Factors: A Single-center Experience from China. Asian Pac J Cancer Prev. 2015;16:4421-4427.

29. Farinati $F$ et al. Diagnostic and prognostic role of alpha-fetoprotein in hepatocellular carcinoma: Both or neither? Am J Gastroenterol. 2006;101:524-532.

30. Abbasi A, Bhutto AR, Butt N, Munir SM. Corelation of serum alpha fetoprotein and tumor size in hepatocellular carcinoma. J Pak Med Assoc. 2012;62:33-36. 